\title{
DITS: un programa informático para el aprendizaje del código dactilológico
}

\author{
José L. Rodríguez Illera
}

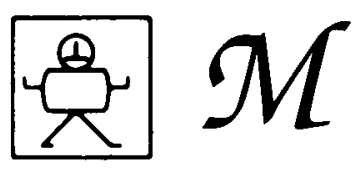

El objetivo de este artículo es describir un programa informático realizado con el propósito de facilitar el aprendizaje del código dactilológico. Este programa debe entenderse en el contexto más general de proporcionar ayudas técnicas a las personas discapacitadas, así como a aquellas que se relacionan con ellas de manera directa, bien sea en contextos escolares, familiares o laborales.

Haremos, en primer lugar, una breve referencia al código dactilológico y su lugar en la comunicación entre personas con discapacidad auditiva, y en segundo lugar, una presentación del programa informático.

\section{EL CÓDIGO DACTILOLÓGICO}

Existe en la actualidad una importante polémica sobre el lugar que ocupan los distintos sistemas de comunicación entre personas con déficit auditivo, así como entre ellas y otras personas no afectadas de limitaciones auditivas. Esta polémica es retrazada al pasado, especialmente a los avatares tanto técnicos como ideológicos, que la enseñanza a personas sordas ha sufrido especialmente en este último siglo.

En tal debate, la dactilología apenas ocupa lugar, desplazada por la cuestión principal de la lengua de signos y de su posición tanto en la enseñanza como en la relación social de las personas sordas. Pero sí que permanece como un resto, ligado a la designación de nombres o palabras nuevos, así como a determinadas categorías nominales - típicamente nombres de personas, de ciudades, etc. Antes de que un gesto nuevo ocupe el lugar para designar, o incluso más allá por tratarse de nombres ocasionales, $o$, simplemente, por la necesidad de posibilitar la comunicación entre personas de ámbitos geográficos distintos, el recurso a "signar/dactilologizar" la palabra se vuelve necesario. Por esa razón es difícil que un código simple, basado en el alfabeto de cada lengua pero muy parecido entre ellas, pueda llegar a no utilizarse.

Por otra parte, en situaciones de comunicación entre personas sordas y oyentes, el recurso a este código resuelve los mismos problemas si no más. Finalmente, muchos de los profesionales que tienen a su cargo niños o adolescentes sordos tienen que utilizarlo forzosamente, así como los propios padres.

En conjunto, el código dactilológico ocupa un lugar secundario en la comunicación entre personas sordas, o sordas y oyentes, pero un lugar necesario y difícilmente prescindible. 


\section{EL PROGRAMA DITS}

La utilización de recursos informáticos para facilitar el aprendizaje parece estar hoy plenamente asumida en algunos campos. Por desgracia, en el caso de la dactilología se plantea un problema de tridimensionalidad que difícilmente puede ser resuelto con ordenadores convencionales ${ }^{1}$. Por ello, este tipo de enfoques presenta una limitación relativa al medio utilizado para enseñar. Sin embargo, presenta otras ventajas importantes: la posibilidad de poder ser utilizado de forma repetitiva, $y$ siempre de manera distinta, la velocidad, la no necesidad de personas que sepan el código para poder transmitirlo, etc. En conjunto, las ventajas e inconvenientes de la enseñanza/ aprendizaje basados en ordenadores.

En el caso del código dactilológico hay algunos aspectos que lo hacen particularmente adecuado para un enfoque de este tipo: se trata de la automatización de una destreza, que no requiere por tanto un grado de conciencia del sujeto sobre el resultado final (aunque sí en el proceso de aprendizaje), lo que permite además el poder utilizar el ordenador para una tarea mecánica. Por otra parte, aunque el alfabeto sea cerrado, su combinatoria es siempre abierta y, potencialmente, "infinita"

Dits se compone de 3 módulos de aprendizaje más un editor de textos simplificado y unas utilidades de personalización.

El primero módulo, o de aprendizaje básico (el adjetivo sugiere que es el lugar mejor para empezar), muestra todo el alfabeto convencional castellano y catalán , permitiendo obtener una traducción inmediata de cualquier letra a su representación dactilográfica. Este módulo permite también escribir palabras en una caja de texto que son dactilografiadas - pudiendo el usuario determinar la velocidad entre letra y letra. El objetivo del módulo es una transacción típica de reconocimiento o identificación. A diferencia de identificaciones más complejas, en el código dactilológico no hay lugar para distinciones del tipo partes-todo, o partes y subpartes, por lo que se trata de un 'simple' reconocimiento visual.

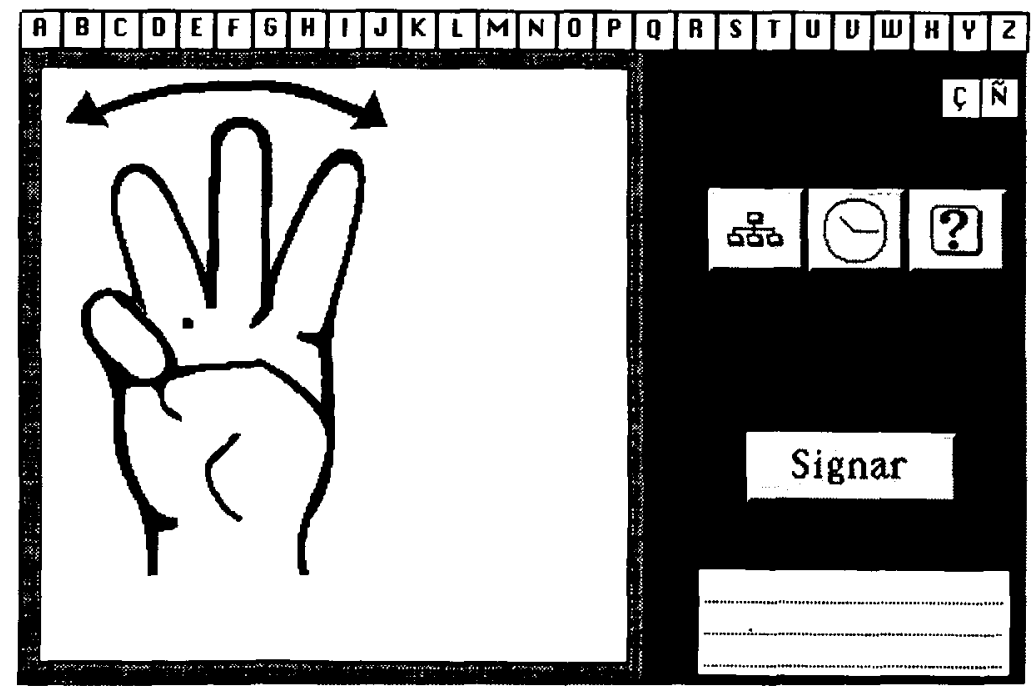

Al igual que en el resto del programa, las letras del alfabeto han sido dibujadas desde la posición del interlocutor y no de la persona que se expresa. Ello es así por considerar que se debe hacer el esfuerzo de adecuar la posición de la mano a aquello que va a ser percibido - además del motivo obvio de la dificultad para percibir ade- 
cuadamente, por parte de la persona que utiliza el código, su propia mano orientada hacia un interlocutor situado en otra(s) posición(es). La consecuencia es que el usuario de Dits puede tener que adecuar constantemente su mano en relación a la imagen que se ve en pantalla, pero ésto parece inevitable en este módulo básico.

El segundo módulo, aprendizaje avanzado, permite una actividad de decodificación. El usuario debe traducir a la lengua de uso activa (catalán o castellano) una palabra completa escrita en dactilología. El ordenador evalúa inmediatamente si la traducción es correcta. En el caso de que se dude, un sistema de ayuda va ofreciendo, letra a letra, la solución.

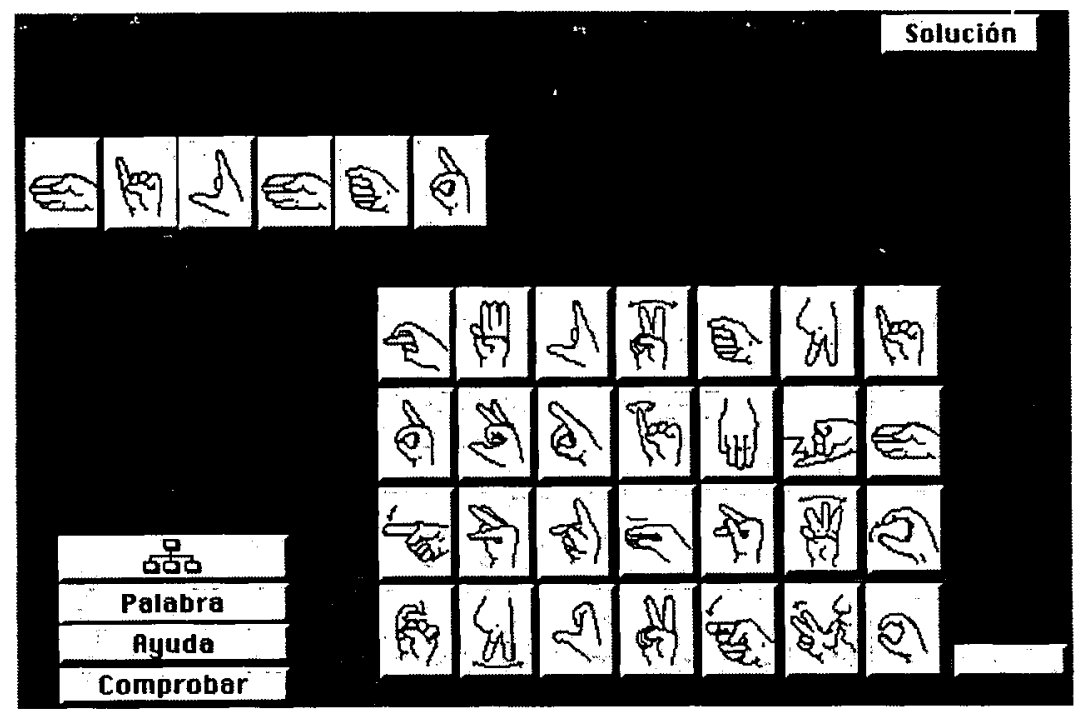

Una segunda posibilidad, en el interior de este módulo, es no ver las palabras escritas sino "signadas", esto es, escritas letra a letra de forma secuencial. Se trata de una opción más compleja, que supone un procesamiento mucho mayor y más rápido de la información, y que no permite ayuda (aunque sí regular la velocidad de presentación de cada letra). El objetivo es reconocimiento en ambos casos. El reconocimiento debe ser escrito en la lengua de uso, por lo que puede tener una utilización inversa (en relación a los objetivos primarios de potenciar el aprendizaje de la dactilología) en el caso de aquellos sujetos sordos que ya conozcan bien el código dactilológico pero que todavía estén afianzado la lengua escrita.

Finalmente, un tercer módulo denominado "Test" ejecuta una simulación de actividad codificativa. Aparece una palabra en la lengua de uso y se tiene que escribir en código dactilográfico. La forma de escribir se hace seleccionando, uno a uno, los signos que aparecen simultáneamente en pantalla. Para evitar pistas de posición, cada vez que se entra en este módulo los signos se redistribuyen al azar. La ayuda consiste en poder ver, durante unos segundos, el alfabeto convencional que "se esconde" detrás de cada signo.

Tanto en el segundo como en el tercer módulo la actividad, sea mediante una transacción de reconocimiento o de producción, consiste en traducir de un código a otro. Para ello se utilizan palabras. Estas palabras están agrupadas en listas, que vienen a funcionar como campos semánticos para el usuario (p.e. la lista de nombres de ciudades, de nombres de personas, etc.), y que se mantienen independientes para los dos idiomas de uso. Estas listas son edirables y configurables por el usuario o por el profesor, de manera que pueden crearse listas específicas 
para un sujeto determinado. Una vez editadas para un idioma, aparecen en las pantallas correspondientes a las módulos y la selección de palabras se realiza, al azar, de la lista elegida.

Finalmente, Dits incorpora un editor de textos muy sencillo, que consta de dos campos en los que se puede escribir texto plano (sin negritas, tipos o tamaños). En uno de los campos se escribe con un tipo de letra convencional, mientras que en el otro se escribe directamente con Dedos - la letra en formato TrueType que contiene el alfabeto dactilológico. Unos botones permiten realizar las dos traducciones posibles de manera sencilla. Aunque es obvio que la utilidad de la dactilología no consiste en traducir textos sino determinados grupos de palabras, el editor se ha incluído pensando en que permita realizar pequeños juegos o actividades con el niño sordo.

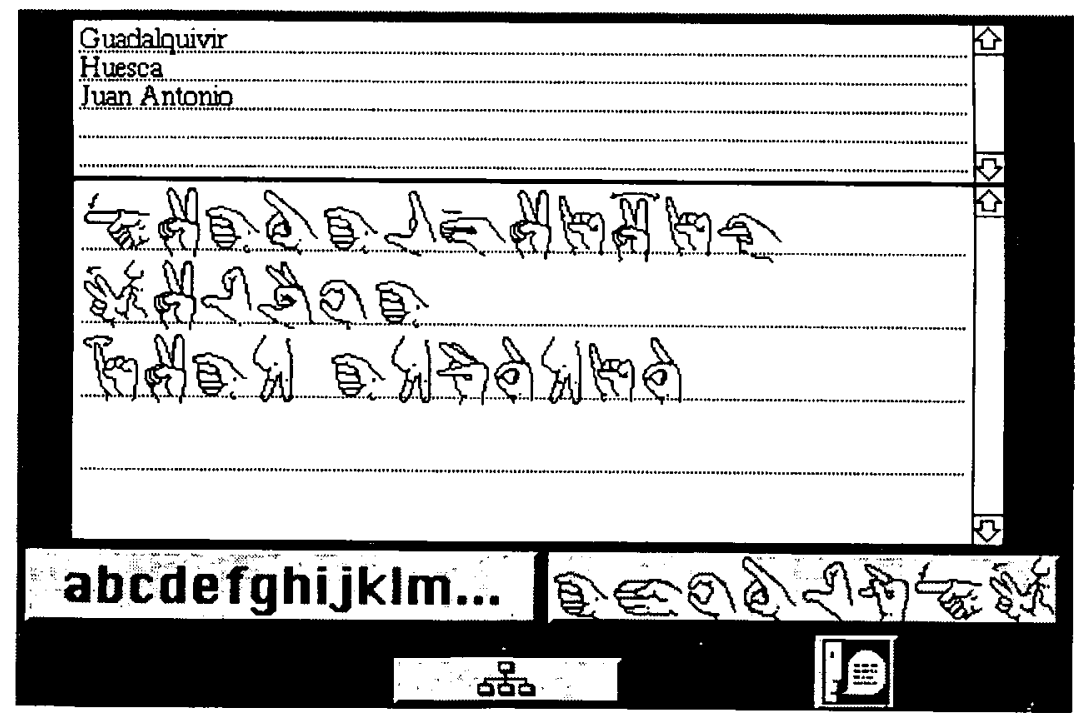

Todas las actividades de Dits, incluído el procesador, están planteadas de una manera similar: es el usuario el que puede elegir libremente, pedir ayuda, o salir de la actividad. En este sentido, se trata de un programa que permite "explorar" las distintas traducciones/equivalencias del código sin la presión que puede suponer un control de la actividad por parte del ordenador. Es de suponer que las personas que lo utilicen tengan una motivación exterior al programa para aprender el código, por lo que no parece aconsejable recurrir a formas instructivas más cerradas - en cualquier caso, éstas pueden aparecer bajo la forma de tener que acertar la traducción en algunos de los módulos.

En resumen, Dits incorpora distintos tipos de actividades orientadas a facilitar el aprendizaje de un código dactilológico. Algunas de las personas que tengan que aprenderlo usarán más unas que otras, y, probablemente, habrá explotaciones de este material en situaciones de reeducación y no sólo de aprendizaje. A ello, esperamos, contribuirá el que se haya hecho de manera abierta, permitiendo la incorporación de listas para situaciones concretas, así como la inclusión de la letra Dedos, que una vez instalada en el ordenador puede ser utilizada con cualquier procesador de textos o programa gráfico, a cualquier tamaño, así como el haberse realizado en versiones para los dos sistemas operativos más difundidos . 


\section{Notas}

1. No así con máquinas de "realidad virtual". Sin embargo, esas máquinas que permiten transmitir una sensación de "espacialidad propioceptiva" fácilmente no parecen estar preparadas todavía para usos de aprendizaje guiado y repetitivo.

2. A excepción de la ele geminada catalana. Esta grafía $(1 \cdot 1)$ presenta problemas importantes para ser reproducida a través de un teclado convencional, dado que es leída como dos letras más un signo de puntuación (en realidad es un punto 'volado'). Algo menos complejo ocurre con la ce con cedilla (ç) que es interpretada como una c especial y con la eñe que es interpretada como una n especial. Ambas letras se han integrado en la ripografía diseñada para Dits.

3. La letra Dedos ha sido diseñada conjuntamente con Josep Martínez Ruzafa, siguiendo el alfabeto expuesto en el libro de J. Perelló y J. Frigola Lenguaje de Signos Manuales. Este alfabeto ha sido retocado en función de sugerencias de profesionales del campo reeducativo, para adecuarse a los usos actuales de algunos signos.

4. Dits se ha realizado en versión para Windows 3.1 o superior y para Macintosh 6.07 o superior.

\section{DITS: un programa informático para el aprendizaje del código dactilológico \\ José L. Rodríguez Illera \\ $C L \& E, 1995,26$}

Resumen: En este artículo se expone los principios de un programa informático denominoado DITS que se ha realizado para aprender dactilología. El programa utiliza un tipo de letra, diseñado especialmente, que reproduce los signos manuales equivalentes al alfabeto castellano y catalán. Dits consta de distintos módulos, de dificultad creciente, así como de un editor de listas de palabras que permite incorporar nuevas a las secuencias de aprendizaje.

Agradecimientos: El autor desea expresar su agradecimiento a Mercedes León, del EAP del CREDAC Pere Barnils de Barcelona, por las ideas para la confección del programa.

Datos sobre el autor: Jose L. Rodriguez Illera es profesor de la Universidad de Barcelona, trabajando en la actualidad en el Instituto de Ciencias de la Educación de la misma como responsable de investigación.

Dirección del autor: Institut de Ciències de l'Educació, Universitat de Barcelona

(1) PERMISOS PARA CITAR O REPRODUCIR EN OTRAS FUENTES: Se pueden citar libremente hasta 500 palabras. Para reproducir una porción de texto mayor, figuras o ilustraciones, se deberá pedir permiso por escrito a la revista, especificando el uso al que se descina el texto. En todos los casos, se deberá citar el copyright de $C L \& E$. En el caso de artículos o textos que hayan sido a su vez reproducidos en $C L \& E$ los interesados deberán dirigirse tanto a los detentadores del copyright original como a $C L \& E$, en el caso de que se quiera hacer uso de la traducción. FOTOCOPIAS: Para todo lo relacionado con el uso mediante fotocopia del material de esta revista, deberán dirigirse a: CEDRO, C/ José Marañón, 10, 3." Izda. Tel. 5941575 . Fax 4453567 